\title{
ON SIMILARITIES BETWEEN EXPONENTIAL POLYNOMIALS AND HERMITE POLYNOMIALS
}

\author{
Edyta Hetmaniok, Mariusz Pleszczyński, Damian Stota, Roman Wituła \\ Institute of Mathematics, Silesian University of Technology \\ Gliwice, Poland \\ damian.slota@polsl.pl
}

\begin{abstract}
The aim of this paper is to introduce and compare some fundamental analytical properties of the title polynomials. Many similarities between them are emphasized in the paper. Moreover, the authors present many isolated results, new proofs and identities.
\end{abstract}

Keywords: Stirling numbers, Bell polynomials, Hermite polynomials, exponential polynomial, generalized Hermite-Bell polynomials, Dobinski's formula

AMS Subject Classification: 11B73, 05A19, $11 C 08$.

\section{Introduction}

Exponential polynomials and generalized Hermite-Bell polynomials were defined by the well-known American mathematician (and intellectualist) E.T. Bell in paper [1].

From the moment of the appearance of these polynomials, their properties inspired many mathematicians to perform investigations. And as the analysis of literature shows, the research is still developing. Moreover, the range of applications of these polynomials is still getting wider - including combinatorics, statistics, theory of orthogonal polynomials, as well as quantum mechanics and probability and random polynomials [2].

The observation of literature also gave us the inspiration for making an attempt of collecting and comparing the properties of considered polynomials. We noticed many similarities between these polynomials, arising mostly from the nature of their generating functions. Differences, usually "slight", between the polynomials exist as well, certainly (for example the problem of binomial type of these polynomials). Moreover, some new relations are received and many original proofs are demonstrated in the paper.

\section{Exponential polynomials}

Exponential polynomials $B_{n}(x), n \in \mathbb{N}$, also called the single variable Bell polynomials, are defined by the formula 


$$
B_{n}(x)=\sum_{\pi} x^{|\pi|}
$$

where $\pi$ ranges over all partitions of $n$-element set and $|\pi|$ denotes the number of blocks in the partition $\pi$ (see [3]). It is obvious that

$$
B_{n}(x)=\sum_{k=1}^{n}\left\{\begin{array}{l}
n \\
k
\end{array}\right\} x^{k}
$$

where $\left\{\begin{array}{l}n \\ k\end{array}\right\}$ denotes the Stirling numbers of the second kind (the Karamata-Knuth notation $[4,5]$ is used here). The above polynomials satisfy the following recurrent relation

$$
B_{n+1}(x)=x\left(B_{n}(x)+\frac{d}{d x} B_{n}(x)\right) .
$$

We shall now prove the following theorems on the generating functions for these polynomials. Although relation (4) presented below is known, still the way in which it was generated here seems to be completely new (see $[1,4,6])$.

Theorem 1. The following relations hold $(x, a \in \mathbb{C})$ :

$$
\begin{aligned}
\frac{d^{n-1}}{d x^{n-1}}\left(e^{x+a e^{x}}\right) & =\sum_{k=1}^{n}\left\{\begin{array}{l}
n \\
k
\end{array}\right\} a^{k-1} e^{k x+a e^{x}}, \\
a e^{-a} e^{x+a e^{x}} & =\sum_{n=0}^{\infty} B_{n+1}(a) \frac{x^{n}}{n !}, \\
e^{a\left(e^{x}-1\right)} & =\sum_{n=0}^{\infty} B_{n}(a) \frac{x^{n}}{n !} .
\end{aligned}
$$

Proof (2): Since $\left\{\begin{array}{l}n \\ k\end{array}\right\} \equiv 1$, equality (2) for $n=1$ holds. Suppose that (2) holds for some positive integer $n$. Because

$$
\left\{\begin{array}{l}
n \\
1
\end{array}\right\} \equiv 1 \quad \text { and } \quad\left\{\begin{array}{c}
n+1 \\
k
\end{array}\right\}=k\left\{\begin{array}{l}
n \\
k
\end{array}\right\}+\left\{\begin{array}{c}
n \\
k-1
\end{array}\right\}
$$

it can easily be verified that 


$$
\begin{gathered}
\frac{d^{n}}{d x^{n}}\left(e^{x+a e^{x}}\right)=\sum_{k=1}^{n}\left\{\begin{array}{l}
n \\
k
\end{array}\right\} a^{k-1} \frac{d}{d x}\left(e^{k x+a e^{x}}\right)= \\
=\sum_{k=1}^{n}\left\{\begin{array}{l}
n \\
k
\end{array}\right\} a^{k-1}\left(k+a e^{x}\right) e^{k x+a e^{x}}= \\
=\left\{\begin{array}{l}
n \\
1
\end{array}\right\} e^{x+a e^{x}}+\sum_{k=2}^{n} a^{k-1}\left(k\left\{\begin{array}{l}
n \\
k
\end{array}\right\}+\left\{\begin{array}{c}
n \\
k-1
\end{array}\right\}\right) e^{k x+a e^{x}}+ \\
+\left\{\begin{array}{l}
n \\
n
\end{array}\right\} a^{n} e^{(n+1) x+a e^{x}}=\sum_{k=1}^{n+1}\left\{\begin{array}{c}
n+1 \\
k
\end{array}\right\} a^{k-1} e^{k x+a e^{x}} .
\end{gathered}
$$

The application of principal of mathematical induction finishes the proof of equality (2).

Proof (3) and (4): From (2) we obtain

$$
a e^{-a}\left(e^{x+a e^{x}}\right)_{x=0}^{(n-1)}=\sum_{k=1}^{n}\left\{\begin{array}{l}
n \\
k
\end{array}\right\} a^{k}=B_{n}(a),
$$

which implies formula (3).

We note that (3) is equivalent to (4), since we have

$$
a \int_{0}^{x} e^{z+a e^{z}} d z=e^{a e^{x}}-e^{a}
$$

i.e.

$$
a e^{-a} \int_{0}^{x} e^{z+a e^{z}} d z=e^{a\left(e^{x}-1\right)}-1
$$

Remark 2. The general formula of type (2) can be found in [7]:

$$
\frac{d^{n}}{d x^{n}} F\left(e^{x}\right)=\sum_{k=1}^{n}\left\{\begin{array}{l}
n \\
k
\end{array}\right\} e^{k x} F^{(k)}\left(e^{x}\right) .
$$

If we substitute $F(t)=t e^{a t}\left(t=e^{x}\right)$ formula (2) can be deduced.

Remark 3. We note that the function

$$
\varphi(t):=e^{a\left(e^{i t}-1\right)}
$$


is the characteristic function of Poisson distribution [8]. By (4), the following formulae could be obtained (see also formulae (19) and (20)):

$$
\frac{1}{2}(\varphi(t)+\varphi(-t))=e^{a(\cos t-1)} \cos (a \sin t)=\sum_{n=0}^{\infty}(-1)^{n} B_{2 n}(a) \frac{t^{2 n}}{(2 n) !}
$$

and

$$
\frac{1}{2 i}(\varphi(t)-\varphi(-t))=e^{a(\cos t-1)} \sin (a \sin t)=\sum_{n=0}^{\infty}(-1)^{n} B_{2 n+1}(a) \frac{t^{2 n+1}}{(2 n+1) !},
$$

which are similar to known Euler's formulae

$$
\cos t=\frac{1}{2}\left(e^{i t}+e^{-i t}\right) \quad \text { and } \quad \sin t=\frac{1}{2 i}\left(e^{i t}-e^{-i t}\right) .
$$

Corollary 4. The following formula of convolution type for $B_{n}(x)$ holds:

$$
B_{n}(a+b)=\sum_{k=0}^{n}\left(\begin{array}{l}
n \\
k
\end{array}\right) B_{k}(a) B_{n-k}(b)
$$

thus, the Bell polynomials are of the binomial type and the whole machinery of the umbral calculus can be applied to $B_{n}(x), n \in \mathbb{N}$ [9-11];

$$
a e^{x}=\sum_{n=0}^{\infty}\left(\sum_{k=0}^{n}\left(\begin{array}{l}
n \\
k
\end{array}\right) B_{k+1}(a) B_{n-k}(-a)\right) \frac{x^{n}}{n !}
$$

which implies

$$
a=\sum_{k=0}^{n}\left(\begin{array}{l}
n \\
k
\end{array}\right) B_{k+1}(a) B_{n-k}(-a)
$$

for every $n \geq 0$. Moreover, we have

$$
e^{a}=\sum_{n=0}^{\infty} \frac{(\ln 2)^{n}}{n !} B_{n}(a)=1+\sum_{k=1}^{\infty}\left(\sum_{n=k}^{\infty}\left\{\begin{array}{l}
n \\
k
\end{array}\right\} \frac{(\ln 2)^{n}}{n !}\right) a^{k} .
$$

Hence, we get

$$
k ! \sum_{n=k}^{\infty}\left\{\begin{array}{l}
n \\
k
\end{array}\right\} \frac{(\ln 2)^{n}}{n !} \equiv 1 .
$$

Our next result also follows from Theorem 1. It is treated as a new kind of reduction formulae for the indices of the Bell polynomials. 
Theorem 5. The following recursion formulae hold

$$
\begin{gathered}
B_{n+1}(a)=a \sum_{k=0}^{n}\left(\begin{array}{l}
n \\
k
\end{array}\right) B_{k}(a), \\
B_{n+2}(a)=a \sum_{k=0}^{n}\left(\left(\begin{array}{c}
n+1 \\
k
\end{array}\right)+a\left(\begin{array}{l}
n \\
k
\end{array}\right)\right) B_{k}(a), \\
B_{n+3}(a)=a \sum_{k=0}^{n}\left(\left(\begin{array}{c}
n+2 \\
k
\end{array}\right)+a\left(\left(\begin{array}{c}
n+2 \\
n+1
\end{array}\right)\left(\begin{array}{l}
n \\
k
\end{array}\right)+\left(\begin{array}{c}
n+1 \\
k
\end{array}\right)\right)+a^{2}\left(\begin{array}{l}
n \\
k
\end{array}\right)\right) B_{k}(a)= \\
\left.=a \sum_{k=0}^{n}\left(\begin{array}{c}
n+2 \\
k
\end{array}\right)+a\left(\begin{array}{c}
n+1 \\
k
\end{array}\right)\left(n+3-k \frac{n+2}{n+1}\right)+a^{2}\left(\begin{array}{l}
n \\
k
\end{array}\right)\right) B_{k}(a)
\end{gathered}
$$

and

$$
\begin{gathered}
B_{n+4}(a)=a \sum_{k=0}^{n}\left(\left(\begin{array}{c}
n+3 \\
k
\end{array}\right)+a\left(\left(\begin{array}{l}
n+3 \\
n+1
\end{array}\right)\left(\begin{array}{l}
n \\
k
\end{array}\right)+\left(\begin{array}{c}
n+3 \\
n+2
\end{array}\right)\left(\begin{array}{c}
n+1 \\
k
\end{array}\right)+\right.\right. \\
\left.\left.+\left(\begin{array}{c}
n+3 \\
n+3
\end{array}\right)\left(\begin{array}{c}
n+2 \\
k
\end{array}\right)\right)+a^{2}\left(\left(\begin{array}{c}
n+1 \\
k
\end{array}\right)+(2 n+5)\left(\begin{array}{l}
n \\
k
\end{array}\right)\right)+a^{3}\left(\begin{array}{l}
n \\
k
\end{array}\right)\right) B_{k}(a) .
\end{gathered}
$$

In other words, for every $m \in \mathbb{N}$ there exist polynomials $\mathcal{A}_{n, m, k} \in \mathbb{N}[a]$, $k=0,1, \ldots, n$, such that

$$
\begin{gathered}
B_{n+m}(a)=a \sum_{k=0}^{n} \mathcal{A}_{n, m, k}(a) B_{k}(a) \\
\mathcal{A}_{n, m, k}(a)=\left(\begin{array}{l}
n \\
k
\end{array}\right) a^{m-1}+\cdots+\left(\sum_{r=1}^{m-1}\left(\begin{array}{c}
n+m-1 \\
n+r
\end{array}\right)\left(\begin{array}{c}
n+r-1 \\
k
\end{array}\right)\right) a+\left(\begin{array}{c}
n+m-1 \\
k
\end{array}\right),
\end{gathered}
$$

where

$$
\mathcal{A}_{n, m+1, k}(a)=\mathcal{A}_{n+1, m, k}(a)+\left(\begin{array}{l}
n \\
k
\end{array}\right) \mathcal{A}_{n, m, n+1}(a),
$$

for $k=0,1, \ldots, n$.

Proof: By (2) and (3) we obtain

$$
\begin{gathered}
\sum_{n=0}^{\infty} B_{n+1}(a) \frac{x^{n}}{n !}=a e^{x} \sum_{n=0}^{\infty} B_{n}(a) \frac{x^{n}}{n !}=\left(\sum_{n=0}^{\infty} a \frac{x^{n}}{n !}\right)\left(\sum_{n=0}^{\infty} B_{n}(a) \frac{x^{n}}{n !}\right)= \\
=\sum_{n=0}^{\infty} \sum_{k=0}^{n} a B_{n-k}(a) \frac{x^{n}}{k !(n-k) !}=\sum_{n=0}^{\infty}\left(a \sum_{k=0}^{n}\left(\begin{array}{l}
n \\
k
\end{array}\right) B_{k}(a)\right) \frac{x^{n}}{n !}
\end{gathered}
$$


which implies (10). Now, from (10) we get

$$
\begin{gathered}
B_{n+2}(a)=a \sum_{k=0}^{n+1}\left(\begin{array}{c}
n+1 \\
k
\end{array}\right) B_{k}(a)=a\left(B_{n+1}(a)+\sum_{k=0}^{n}\left(\begin{array}{c}
n+1 \\
k
\end{array}\right) B_{k}(a)\right)= \\
\stackrel{(10)}{=} a \sum_{k=0}^{n}\left(\left(\begin{array}{c}
n+1 \\
k
\end{array}\right)+a\left(\begin{array}{l}
n \\
k
\end{array}\right)\right) B_{k}(a)
\end{gathered}
$$

which gives formula (11). Similarly, the other formulae can be generated.

Remark 6. The above recursion formulae are an alternative to other known formulae of this type:

- Radoux formula [12]:

$$
B_{m+n}(x)=\sum_{k=0}^{\min \{m, n\}} \frac{x^{k}}{k !}\left(\frac{d^{k}}{d x^{k}} B_{m}(x)\right)\left(\frac{d^{k}}{d x^{k}} B_{n}(x)\right) ;
$$

- Spivey-Gould-Quaintance formulae [13]:

$$
\sum_{m=0}^{p}\left[\begin{array}{l}
p \\
m
\end{array}\right] B_{m+n}(x)=x^{p} \sum_{k=0}^{n}\left(\begin{array}{l}
n \\
k
\end{array}\right) p^{k} B_{n-k}(x),
$$

where $\left[\begin{array}{l}p \\ m\end{array}\right]$ denotes the Stirling numbers of the first kind (see $\left.[4,5]\right)$, and

$$
B_{m+n}(x)=\sum_{j=0}^{m} \sum_{k=0}^{n}\left\{\begin{array}{l}
m \\
j
\end{array}\right\}\left(\begin{array}{l}
n \\
k
\end{array}\right) j^{n-k} x^{j} B_{k}(x) ;
$$

- Cigler formula (and their q-analogue) [14]:

$$
\sum_{k=1}^{n}(-1)^{n-k}\left[\begin{array}{l}
n \\
k
\end{array}\right] B_{k+h}(x)=x^{n} \sum_{j=0}^{h}\left(\begin{array}{l}
h \\
j
\end{array}\right) n^{h-j} B_{j}(x) .
$$

Remark 7. By applying the following differential operator $\Theta=x \frac{d}{d x} n$-times to $e^{x}$, we obtain

$$
\Theta^{n} e^{x}=\sum_{k=1}^{\infty} \frac{k^{n} x^{k}}{k !}
$$

and, simultaneously, the formula

$$
\Theta^{n} e^{x}=e^{x} B_{n}(x)
$$


which implies the generalized Dobinski's formula (see [15]):

$$
B_{n}(x)=e^{-x} \sum_{k=1}^{\infty} \frac{k^{n} x^{k}}{k !}
$$

Harper $(1967,[16])$ showed that polynomials $B_{n}(x), n \in \mathbb{N}$, have only real simple zeros (see also [17] where some weaker result is discussed). We note that Cakic in papers $[18,19]$ studied some combinatorial properties of the generalizations of the above $\Theta$-differential operator.

\section{Hermite polynomials}

There exist some equivalents to many facts and identities from Section 1 for the Hermite polynomials

$$
H_{n}(x)=(-1)^{n} e^{x^{2}} \frac{d^{n}}{d x^{n}}\left(e^{-x^{2}}\right)=\sum_{k=0}^{\lfloor n / 2\rfloor} \frac{(-1)^{k} n !}{k !(n-2 k) !}(2 x)^{n-2 k}
$$

We have:

- equivalent of (1) (see [20]):

$$
H_{n+1}(x)=2 x H_{n}(x)-\frac{d}{d x} H_{n}(x) ;
$$

- Dobinski's formula for the Hermite polynomials

$$
H_{n}(x)=(-1)^{n} e^{x^{2}} \sum_{k=\lfloor n / 2\rfloor}^{\infty} \frac{(-1)^{k}(2 k-n+1)_{n}}{k !} x^{2 k-n},
$$

since

$$
\frac{d^{n}}{d x^{n}}\left(e^{-x^{2}}\right)=\frac{d^{n}}{d x^{n}}\left(\sum_{k=0}^{\infty}(-1)^{k} \frac{x^{2 k}}{k !}\right)=\sum_{k=\lfloor n / 2\rfloor}^{\infty}(-1)^{k} \frac{(2 k-n+1)_{n}}{k !} x^{2 k-n}
$$

where $(t)_{n}$ denotes the Pochhammer symbol (i.e. $(t)_{0}:=1,(t)_{n}:=t(t+$ +1) $\ldots(t+n-1)$ for every $n=1,2, \ldots)$;

- in view of (17) and Theorem 2 from paper [21] (see also [22, 23]) all the roots of polynomials $H_{n}(x)$ are real and, what is more, the roots of polynomial $H_{n}(x)$ separate the roots of polynomial $H_{n+1}(x)$ for each $n \in \mathbb{N}$;

- it is deduced from the Schur criterion that $H_{2 n}(x)$ and $H_{2 n-1}(x) / x, n \in \mathbb{N}$, are irreducible polynomials (see [24]); 
- in view of the following reduction identity (see (7)):

$$
H_{n}(x+y)+2^{n-2\lfloor n / 2\rfloor}\left(\begin{array}{c}
n \\
\lfloor n / 2\rfloor
\end{array}\right)(2 x)^{n-2\lfloor n / 2\rfloor}=\sum_{k=0}^{n}\left(\begin{array}{l}
n \\
k
\end{array}\right) H_{n}(x) H_{n}(y),
$$

the Hermite polynomials are not of binomial type. Moreover, there are analogues of (5) and (6):

$$
\begin{gathered}
e^{t^{2}} \cos (2 a t)=\sum_{n=0}^{\infty}(-1)^{n} H_{2 n}(a) \frac{t^{2 n}}{(2 n) !} \\
e^{t^{2}} \sin (2 a t)=\sum_{n=0}^{\infty}(-1)^{n} H_{2 n+1}(a) \frac{t^{2 n+1}}{(2 n+1) !}
\end{gathered}
$$

\section{Generalized Hermite-Bell polynomials}

Many properties of the Hermite polynomials can be easily transformed to the generalized Hermite-Bell polynomials which are defined by the relation

$$
H_{n}^{r}(x)=(-1)^{n} \exp \left(x^{r}\right) \frac{d^{n}}{d x^{n}} \exp \left(-x^{r}\right), \quad n=0,1,2, \ldots,
$$

for every $r=2,3, \ldots$ (this relation could be also discussed for all positive reals $r$ ).

We note that polynomials $H_{n}^{r}(x)$ are a special case of the polynomials

$$
\xi_{n}(p, x ; r):=\exp \left(-p x^{r}\right) \frac{d^{n}}{d x^{n}} \exp \left(p x^{r}\right)
$$

introduced by E.T. Bell in [1], since

$$
H_{n}^{r}(x)=(-1)^{n} \xi_{n}(-1, x ; r) .
$$

Moreover, the polynomials $H_{n}^{2}(x)$ are our classical Hermite polynomials $H_{n}(x)$ discussed in Section 2. Quite important fact is that the polynomials $H_{n}^{r}(x)$ for even positive integers $r>2$ do not form an orthogonal set with respect to the weight function $\exp \left(-x^{r}\right)$ on the interval $(-\infty, \infty)[25]$.

Now a collection of the basic properties of polynomials $H_{n}^{r}(x)$ will be listed. First one will be the following differential-difference equation (see (17)):

$$
H_{n+1}^{r}(x)=r x^{r-1} H_{n}^{r}(x)-\frac{d}{d x} H_{n}^{r}(x) .
$$


Second one, the Dobinski's formula (see (18)):

$$
H_{n}^{r}(x)=(-1)^{n} \exp \left(x^{r}\right) \sum_{k=\lfloor n / r\rfloor}^{\infty} \frac{(-1)^{k}}{k !}(r k-n+1)_{n}
$$

for every positive real number $r$. Next, the generating function for $H_{n}^{r}(x)$ (see [26]):

$$
\exp \left(x^{r}-(x-\tau)^{r}\right)=\sum_{n=0}^{\infty} H_{n}^{r}(x) \frac{\tau^{n}}{n !} .
$$

This relation can be easily used for obtaining many new properties of polynomials $H_{n}^{r}(x)$. For example, let $\omega \in \mathbb{C}, \omega^{r}=1$, then

$$
\exp \left(x^{r}-(x-\tau)^{r}\right)=\exp \left((\omega x)^{r}-(\omega x-\omega \tau)^{r}\right),
$$

i.e.

$$
\sum_{n=0}^{\infty} H_{n}^{r}(x) \frac{\tau^{n}}{n !}=\sum_{n=0}^{\infty} H_{n}^{r}(\omega x) \frac{\omega^{n} \tau^{n}}{n !}
$$

which implies

$$
H_{n}^{r}(x)=\omega^{n} H_{n}^{r}(\omega x) .
$$

If we take in (24) $\tau:=x\left(1+e^{i 2 \pi / r}\right)=2 x e^{i \pi / r} \cos (\pi / r)$, then we get

$$
\exp \left(x^{r}-(-x)^{r}\right)=\sum_{n=0}^{\infty} H_{n}^{r}(x) \frac{(2 x \cos (\pi / r))^{n}}{n !} \exp (i n \pi / r),
$$

i.e.

$$
\sum_{n=0}^{\infty} H_{n}^{r}(x) \frac{(2 x \cos (\pi / r))^{n}}{n !} \cos \left(n \frac{\pi}{r}\right)= \begin{cases}1, & \text { for even } r \\ \exp \left(2 x^{r}\right), & \text { for odd } r,\end{cases}
$$

and

$$
\sum_{n=0}^{\infty} H_{n}^{r}(x) \frac{(2 x \cos (\pi / r))^{n}}{n !} \sin (n \pi / r)=0 .
$$

Hence, for the special values of $r$ we obtain:

- from (27) for $r=3$ :

$$
0=\sum_{n=0}^{\infty}(-1)^{n}\left(H_{3 n+1}^{3}(x) \frac{x^{3 n+1}}{(3 n+1) !}+H_{3 n+2}^{3}(x) \frac{x^{3 n+2}}{(3 n+2) !}\right)
$$


- from (27) for $r=4$ :

$$
0=\sum_{n=0}^{\infty}(-4)^{n}\left(\frac{1}{2} H_{4 n+1}^{4}(x) \frac{x^{4 n+1}}{(4 n+1) !}+H_{4 n+2}^{4}(x) \frac{x^{4 n+2}}{(4 n+2) !}+H_{4 n+3}^{4}(x) \frac{x^{4 n+3}}{(4 n+3) !}\right)
$$

- from (26) for $r=4$ :

$$
-x H_{1}^{4}(x)=\sum_{n=1}^{\infty}(-4)^{n}\left(\frac{1}{2} H_{4 n-1}^{4}(x) \frac{x^{4 n-1}}{(4 n-1) !}+H_{4 n}^{4}(x) \frac{x^{4 n}}{(4 n) !}+H_{4 n+1}^{4}(x) \frac{x^{4 n+1}}{(4 n+1) !}\right) .
$$

Let us also notice that from (24) it results that, similarly as the classical Hermite polynomials $H_{n}(x)$, also the polynomials $H_{n}^{r}(x), n=0,1, \ldots$, are not of the binomial type.

Indeed, we have

$$
\exp \left(x^{r}+y^{r}-(x-\tau)^{r}-(y-\tau)^{r}\right)=\sum_{n=0}^{\infty}\left(\sum_{k=0}^{n}\left(\begin{array}{l}
n \\
k
\end{array}\right) H_{k}^{r}(x) H_{n-k}^{r}(y)\right) \frac{\tau^{n}}{n !}
$$

and

$$
\exp \left((x+y)^{r}-(x+y-\tau)^{r}\right)=\sum_{n=0}^{\infty} H_{n}^{r}(x+y) \frac{\tau^{n}}{n !}
$$

as well as, what is easy to verify, for every $r, \tau \in \mathbb{R}, r>0, \tau \neq 0$, the functions

$$
(0, \infty)^{2} \ni(x, y) \mapsto x^{r}+y^{r}-(x-\tau)^{r}-(y-\tau)^{r},
$$

and

$$
(0, \infty)^{2} \ni(x, y) \mapsto(x+y)^{r}-(x+y-\tau)^{r}
$$

are different.

Considering the zeros of polynomial $H_{n}^{r}(x)$ let us notice that

$$
\begin{aligned}
& H_{0}^{r}(x)=1, \\
& H_{1}^{r}(x)=r x^{r-1}, \\
& H_{2}^{r}(x)=x^{r-2}\left(r^{2} x^{r}-r(r-1)\right), \\
& H_{3}^{r}(x)=x^{r-3}\left(r^{3} x^{2 r}-3 r^{2}(r-1) x^{r}+r(r-1)(r-2)\right)
\end{aligned}
$$

and in general $[1,26]$ : 


$$
H_{n}^{r}(x)=x^{r-s} \sum_{k=0}^{N}(-1)^{k} a_{k} x^{(N-k) r},
$$

where $n=q r+s, \quad 1 \leq s \leq r, q \geq 0$ and $s$ are integers, $N=n-q-1$, $a_{k}=a_{k}(n, r)$ are all positive integers. Observe that $H_{2}^{r}(x)$ possesses at most two real zeros, $H_{3}^{r}(x)$ for $r>2$ possesses two or four real zeros, etc. Furthermore, in paper [27] the asymptotic approximation for distribution of the extreme positive real zeros of $H_{n}^{r}(x)$ is given.

\section{References}

[1] Bell E.T., Exponential polynomials, Ann. Math. 1934, 35(2), 258-277.

[2] Nadarajah S., Simple formulas for certain polynomials, Appl. Math. Comput. 2007, 187, 1592$-1596$.

[3] Ehrenborg R., The Hankel determinant of exponential polynomials, Amer. Math. Monthly 2000, 107, 557-560.

[4] Graham R.L., Knuth D.E., Patashnik O., Concrete Mathematics, Addison-Wesley, Reading 1994.

[5] Knuth D.E., Stirling numbers, Amer. Math. Monthly 1995, 102, 562.

[6] Gould H.W., Quaintance J., A linear binomial recurrence and the Bell numbers and polynomials, Appl. Anal. Discrete Math. 2007, 1, 371-385.

[7] Comtet L., Advanced Combinatorics, D. Riedel, Dordrecht 1974.

[8] Lukacs E., Characteristic Functions, Hafner Publishing Co., New York 1970.

[9] Di Bucchianico A., Loeb D., A selected survey of umbral calculus, Electron. J. Combin., Dynamic Surveys 2000, DS3.

[10] Gessel I.M., Applications of the classical umbral calculus, Algebra Universalis 2003, 49, 397-434 .

[11] Roman S.M., Rota G.C., The umbral calculus, Advances in Math. 1978, 27, 95-188.

[12] Radoux Ch., The Henkel determinant of exponential polynomials: a very short proof a new result concerning Euler numbers, Amer. Math. Monthly 2002, 109, 277-278.

[13] Gould H.W., Quaintance J., Implications of Spivey's Bell number formula, J. Integer Sequences 2008, 11, Article 08.3.7.

[14] Fekete A., Cigler J., Stirling and Bell and binomial, Oh my! Amer. Math. Monthly 2002, 109, 476-477.

[15] El-Mikkawy M., A note on the reducibility of special infinite series, Appl. Math. Comput. 2005, 162, 311-315.

[16] Harper L.H., Stirling behavior is asymptotically normal, Ann. Math. Stat. 1967, 38, 401-414.

[17] Brillhart J., Note on the single variable Bell polynomials. Amer. Math. Monthly 1967, 74, 695-696.

[18] Cakić N.P., On some combinatorial identities, Univ. Beograd. Publ. Elektrotehn. Fak. Ser. Mat. Fiz. 1980, 678-715, 91-94.

[19] Cakić N.P., On the numbers related to the Stirling numbers of the second kind, Facta Univ. Ser. Math. Inform. 2007, 22 , 105-108.

[20] Suetin P.K., Classical Orthogonal Polynomials, Nauka, Moscow 1976 (in Russian). 
[21] Ma S.-M., Wang Y., q-Eulerian polynomials and polynomials with only real zeros, Electron. J. Combin. 2008, 15, \#R17.

[22] Liu L., Wang Y., A unified approach to polynomial sequences with only real zeros, Adv. in Appl. Math. 2007, 38, 542-560.

[23] Olver F.W.J., Lozier D.W., Boisvert R.F., Clark C.W. (eds.), NIST Handbook of Mathematical Functions, Cambridge University Press, Cambridge 2010.

[24] Dorwart H.L., Irreducibility of polynomials, Amer. Math. Monthly 1935, 42, 369-381.

[25] Kalinowski M.W., Seweryński M., Differential equation for Hermite-Bell polynomials, Math. Proc. Phil. Soc. 1982, 91(2), 259-265.

[26] Dominici D., Asymptotic analysis of generalized Hermite polynomials, Analysis 2008, 28, 239-261.

[27] Paris R.B., The asymptotics of the generalized Hermite-Bell polynomials, J. Comp. Appl. Math. 2009, 232, 216-226. 